\title{
An accurate method for the extraction of line structured light stripe
}

\author{
Jiwu Wang ${ }^{1}$ \\ School of Mechanical, Electronic and Control Engineering, Beijing Jiaotong University \\ Yaodong Li ${ }^{1}$, Zhijing Jian ${ }^{1}$ \\ School of Mechanical, Electronic and Control Engineering, Beijing Jiaotong University
}

\author{
Masanori Sugisaka ${ }^{2}$ \\ Alife Robotics Corporation Ltd, Japan and Open University, United Kingdom \\ E-mail: jwwang@bjtu.edu.cn,icarob@alife-robotics.co.jp \\ www.bjtu.edu.cn
}

\begin{abstract}
In order to improve the accuracy in the On-line measurement of rail profile with a line structured light based on machine vision, the accurate extraction of a structured light stripe is a necessary and key step. An accurate extraction method is proposed for the structural light stripe in practical applications. The structured light stripe is separated and extracted accurately based on the geometric characteristics of the structure light stripe in the binary image. And the noise in the captured image is removed with region segmentation method. The above method was tested in laboratory conditions. Experiment results show that the developed method can effectively solve the problem of the accurate extraction of structured light stripe in real time.
\end{abstract}

Keywords: Line structured light; region segmentation; Rail profile; Machine vision

\section{Introduction}

With the rapid development of rail traffic, the issues of train safety are becoming more prominent than before. Here railroad plays an important role. Identifying railwear status is critical to keep high-speed running safety. In order to measure the rail profile efficiently, the Online measurement of rail profile with a line structured light based on machine vision is proposed in this paper. The basic measurement principle is that the structured light projected on the object's surface, is captured by a camera, and the modulated strips images record the three-dimensional information about that object surface. In rail profile measurement the structure-light will be distorted when it is projected on rail profile, and this represents the railway profile information. Then the capture image is processed to extract the contour line and calculate its real dimensions. By comparing the calculated results with the standard rail profile, the railway working status will be determined.

In order to ensure the accuracy of measurement, the accurate extraction of a structured light stripe is a key step. The traditional methods ${ }^{1}$ are the threshold method and the extreme value method. Those methods are suitable in the situation when the distinction of grayscale is very large between the background and target area in the capture image. If there are a lot of noises in the image or the grayscale of the target is similar to that of the background, it is not effect for these methods.

In light of above problems, an accurate method used for the extraction of structured light stripe is proposed in this paper. Firstly, horizontal integral projecting and vertical integral projecting is calculated to determine the 
position of target stripe in the image. After that, the most of noise with more pixels in the captured image will be removed with region segmentation method. However, for some little noise with only few pixels, it is needed to consider the characteristics of the stripe, such as the shape, the size and the grayscale gradient. According to these geometric and lumination characteristics of the structure light stripe, the target is extracted from the captured image. The experiments show it can provide more accurate calculated results.

\section{Measurement method}

The traditional rail measurement generally is a contact measurement with a compound tool. It can only be used for the measurement in a specified area. And it is impossible to get the general geometric data of the whole rail. Many conclusions are made by the operator's experience.

The on line measurement can be perform by the noncontact measurement with a line laser using machine vision. In order to get the same measurement as the contact method, it is necessary to determine the measurement base point dynamically. Here a special measurement device is designed to realize measurement automatically as in figure 1 . There are two measurement modules $\mathrm{A}$ and $\mathrm{B}$. Module $\mathrm{A}$ is used for the rail profile measurement, and $\mathrm{B}$ is for determining the base point of that profile. Each of the measurement modules is composed of a line structured light emitter and an industrial camera. Based on the triangular measurement principle, the stripe of the light of the rail profile is transformed to the real dimension. Therefore, the precision of the laser line extraction is important to the final measurement results.

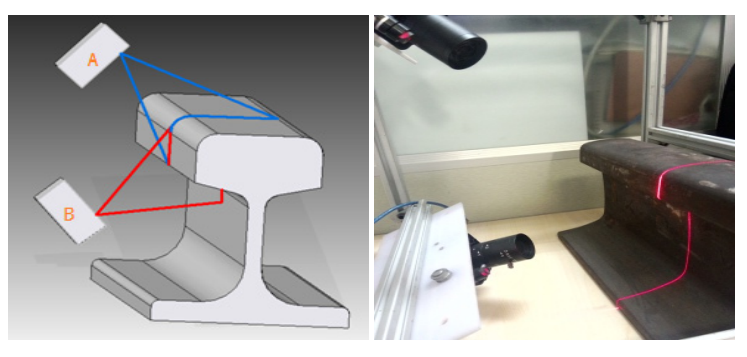

Fig.1.Illustration of the measurement method

\section{Image enhancement}

The on-line measurement of the rail profile is performed in the out-door environment. The illumination conditions generally will vary very fast and irregularly when the vehicle moving. It will influence the quality of the captured images. In order to get reliable image processing results, it is necessary to reduce the illumination influence efficiently. Here the following method is used. Firstly, some high quality images with a laser line are captured as a sample in the laboratory conditions, and the average gray-value of the images is calculated as $g_{-} v a l_{\mathrm{m}}$. It is used as the standard value. In the later measurement process, the average gray-value of each captured image is calculated as $g_{-}$val. The difference between the standard value and is calculated as $\triangle$ val. Finally, the new gray value of each pixel in that image is calculated by subtracted the $\Delta v a l$ with their original gray scale.

The next step is to separate the target area and nontarget area. Horizontal integral projecting and vertical integral projecting is calculated first. According to the variance of the integral value, the target stripe and background are determined. According to the principle of Michelson contrast ${ }^{2}$, the area is extracted as in figure 2. The equation is Eq. (1).

$$
M L C_{(\mathrm{x}, \mathrm{y})}=\frac{I_{(x, y)}-\mu}{I_{(x, y)}+\mu}
$$

$I_{(x, y)}$ is for the gray-value of each pixel, $\mu$ is for the average gray-value of the region.

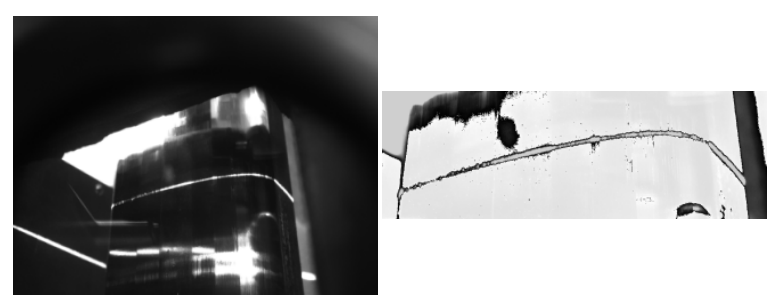

Fig 2.Processing with image enhancement

As shown in Fig.2, by image enhancement, the left image captured outside with much sunshine influence, the laser line is extracted effectively.

\section{Stripe extraction}

With the image enhancement, the influence of illumination is removed, but some noises are left in the image. In order to extract the rail profile stripe efficiently, the noises are removed with following steps. The region's intelligent segmentation is carried on first to remove the most noises of the image. Then the position of the target stripe can be extracted reliably 
with the geometry feature model identification algorithm. Moreover, it can accurately locate the position of the target stripe.

\subsection{Regional intelligent segmentation}

In the rail profile measurement with line structure light, the image with the line structure light contour, as shown in Fig.3, is captured. After the image binarization like in Fig.4, the stripe is in the different connection area separated $^{3}$ with other big noises. Because they occupy different independent regions, it is appropriate to extract them with segmentation method. After this operation, the line structured light contour and a small part as the noise are left, as shown in Fig.5.

By the intelligent segmentation, most noises can be eliminated automatically and efficiently in each captured image. Then the target stripe can be extracted with the previous processing results.

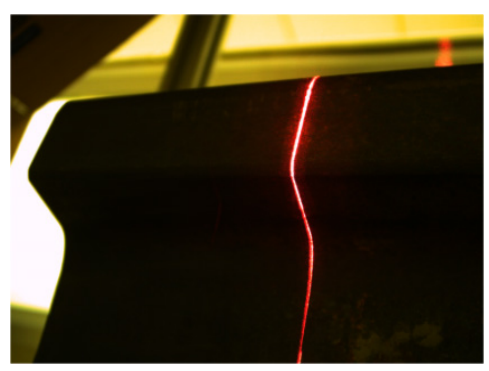

Fig.3. Structured light profile

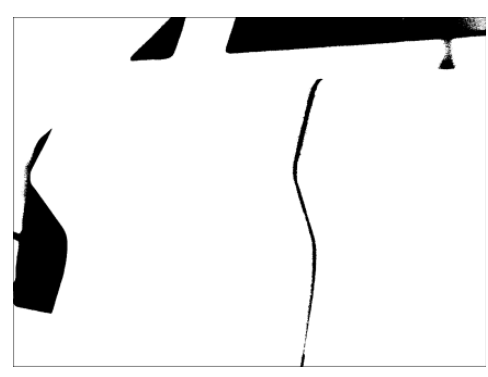

Fig.4.Binary image with image processing

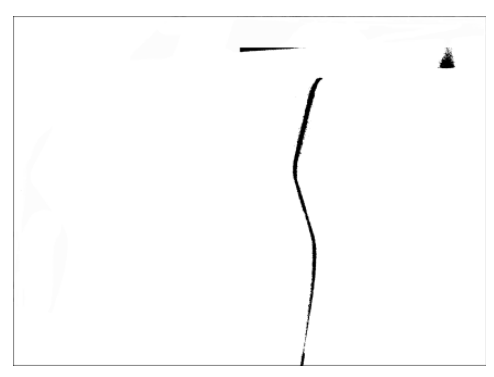

Fig.5. Remove the noise with region segment

\subsection{Identify geometric feature}

Considering the stripe geometric characters, in order to extract the stripe accurately, the structural light is treated as one object. In the boundaries of the stripe, the gray value of each pixel is not zero. And the geometric of the structured light area is different from other noises. It can be segmented with pattern recognition algorithms directly. Then with the continuous scanning of each pixel in the $\mathrm{X}$ direction and the $\mathrm{Y}$ direction respectively, the outer boundary and inner boundary of the line structured light stripe can be accurately extracted ${ }^{4}$. As shown in Fig.6, two continuous boundaries are obtained. With the high resolution of the camera, they accurately represent the geometric feature of the measured rail profile.

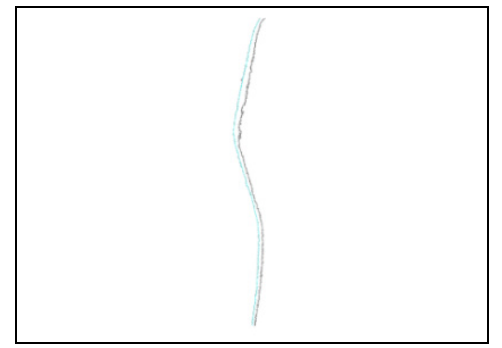

Fig.6. Stripe extraction results

\section{Skeleton extraction}

As shown in Fig.3, the structure light stripe is not a line with one pixel width. The rail profile is represented finally with the one pixel width line. It is necessary to transform the laser line into a line with one pixel width before the real dimension calculation. Here the skeleton algorithm is used after extracting the contour. The normal direction is calculated with the change of the curvature. In this direction, the average coordinate value of each internal and external contour's pixel is calculated as the pixel coordinate of the center stripe ${ }^{5}$. In this experiment, the laser stripe is about the width with 10-20 pixels. After the skeleton processing, the width of the stripe is one pixel in the center of the stripe, so it can improve the accuracy of later transformation. The result of skeleton processing is shown in Fig.7. 


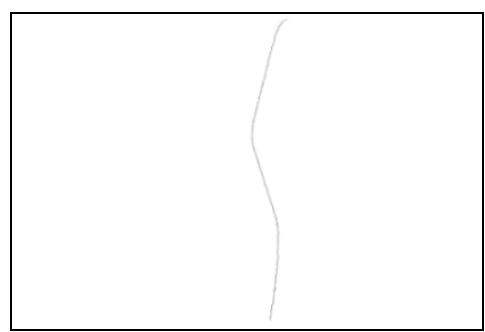

Fig.7. Skeleton processing result

\section{Analysis}

The outer edge and inner edge of the structured light stripe are extracted respectively as shown in the Fig.6. Considering the influence of line laser width in the captured image, the center line is used as the line structured light stripe, as shown in Fig.7. Compared with the outer edge and inner edge, the center line is better to represent the rail profile.

In order to verify the segmentation method in this paper, other edge detection algorithms, such as Roberts operator and Sobel operator, are tested with the same image. Their results are shown in Fig.8. In their result, there are not only the stripe edges but also the internal noise of the stripe. And some of the stripe edges are not composed with a single pixel, so it will influence the measurement accuracy.

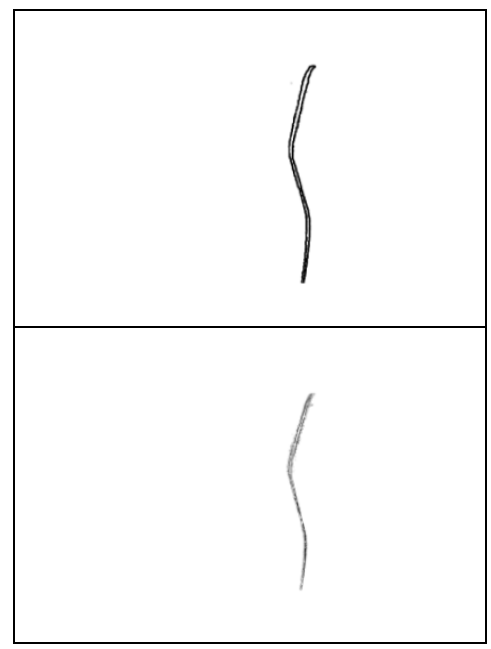

Fig.8. Edge detection operator with Roberts and Sobel

Compared with other image segmentation method, the method used in this paper can effectively eliminate the noise and extract the stripe accurately. With the skeleton algorithm, the central stripe with one pixel width is used as the rail profile for dimension transformation.

\section{Conclusions}

In this paper, based on non-contact measurement with machine vision, a rail profile detection method is given. Due to the complexity in the practical working environment, the image enhancement technique is used to weaken the influence of illumination of outside sun shine. With the region segmentation and pattern recognition of geometric feature, the line structured light stripe is extracted accurately. At the same time, the image processing results are compared with the other edge detection methods, such as Roberts and Sobel. The method used in this paper, can effectively eliminate the noise influence the measurement accuracy ${ }^{6}$.

\section{References}

1. Qiucheng Sun and Renyun Liu, An extraction method of laser stripe centre based on Legendre moment, International Journal for Light and Electron Optics, Volume 127, Issue 2, January 2016, Pages 912-915.

2. Qingyong $\mathrm{Li}$ and Shengwei Ren, A visual detection system for rail surface defects, IEEE Journals \& Magazines, Volume 42, Issue 6, 2012, Pages 1531-1542.

3. Qiucheng Sun and Jian Chen, A robust method to extract a laser stripe centre based on grey level moment, Optics and Lasers in Engineering, Volume 67, April 2015, Pages 122-127.

4. J. Cheng, S.G. Zheng, X.Y. Wu, Structured light-based shape measurement system, Signal Process, 93 (6) (2013), pp. $1435-1444$.

5. Chen Xuanzhen and Wang Xicong, Color structure-light stripe center-line extraction method research, IEEE Conference Publications, 34, July 2015,Pages 3686-3689.

6. Sun J, Zhang J, Liu Z, et al. A vision measurement model of laser displacement sensor and its calibration method. Optics \& Lasers in Engineering, 2013, 51(12):1344-1352. 